\title{
Stretchable and Hydrophobic Electrochromic Devices Using Wrinkled Graphene and PEDOT:PSS
}

\author{
Srinivasa Kartik Nemani, ${ }^{1}$ Dayong Chen, ${ }^{2}$ Marwan H. Mohamed, ${ }^{1}$ and Hossein Sojoudi ${ }^{1}$ \\ ${ }^{1}$ Department of Mechanical, Industrial and Manufacturing Engineering, University of Toledo, Toledo, OH 43606, USA \\ ${ }^{2}$ Department of Mechanical and Aerospace Engineering, University of California, Los Angeles, Los Angeles, CA 90095, USA \\ Correspondence should be addressed to Hossein Sojoudi; hossein.sojoudi@utoledo.edu
}

Received 23 November 2017; Accepted 8 February 2018; Published 22 March 2018

Academic Editor: Bhanu P. Singh

Copyright (c) 2018 Srinivasa Kartik Nemani et al. This is an open access article distributed under the Creative Commons Attribution License, which permits unrestricted use, distribution, and reproduction in any medium, provided the original work is properly cited.

\begin{abstract}
We present an electrochromic device (ECD) fabricated using PEDOT:PSS and graphene as active conductive electrode films and a flexible compliant polyurethane substrate with 1-ethyl-3-methylimidazolium bis(trifluoromethylsulfonyl)imide (EMI-TSFI) additive, as ionic medium. This device with a docile, elastic intermediate substrate along with a transparency controlled PEDOT:PSS film provides a wide color contrast and fast switching rate. We harness wrinkling instability of graphene to achieve a hydrophobic nature without compromising transparency of the ECD. This mechanical self-assembly approach helps in controlling the wavelength of wrinkles generated by inducing measured prestrain conditions and regulating the modulus contrast by selection of underlying materials used, hereby controlling the extent of transparency. The reduction and oxidation switching times for the device were analyzed to be $5.76 \mathrm{~s}$ and $5.34 \mathrm{~s}$ for a $90 \%$ transmittance change at an operating DC voltage of $15 \pm 0.1 \mathrm{~V}$. Strain dependent studies show that the performance was robust with the device retaining switching contrasts even at $15 \%$ uniaxial strain conditions. Our device also exhibits superior antiwetting properties with an average water contact angle of $110^{\circ} \pm 2^{\circ}$ at an induced radial prestrain of $30 \%$ in the graphene film. A wide range color contrast, flexibility, and antiwetting nature of the device envision its uses in smart windows, visors, and other wearable equipment where these functionalities are of outmost importance for developing new generation of smart interactive devices.
\end{abstract}

\section{Introduction}

Electrochromic devices have gained widespread attention over the past decade due to their robust design, ease of manufacturing, and energy saving low power operation mechanisms [1]. Likewise, traditional materials like transition metal oxides and phthalocyanines of rare earth metals like actinides and lanthanides [2] have been largely exploited for exhibiting electrochromic properties in devices. At present, indium tin oxide (ITO) is a widely used transparent conductive electrode in electronics and organic photovoltaics (OPVs) [3-5]. The cost of manufacturing of indium as a rare earth metal rises invariably with depleting resources. Moreover, deposition of ITO on flexible substrates is challenging as it increases its defect densities due to ambient temperature deposition methods and greatly reduces its carrier concentration [6]. While the concept of electrochromic switching has been reported as early as the 1970s [7], nowadays research is more focused on developing organic polymers [8-10] which exhibit similar functionality and properties when compared to their metallic counterparts while being more viable, sustainable, and environmentally friendly [11]. PEDOT:PSS (poly(3,4ethylenedioxythiophene) polystyrene sulfonate) is an organic polymer from the family of thiophenes that has shown very promising results owing to its enhanced conductivity, higher environmental and electrochemical stability, and its conformity as a water-processable polymer [12] leading to extensive research in the field of flexible electronics and as an alternative to conventional metal oxide based electrochromic systems $[13,14]$. Electrochromic contrast is an important parameter in the performance evaluation of such systems. PEDOT:PSS provides high optical contrasts between its redox states [12] enabling this monochromatic transition between colored and bleached states useful in developing absorption and transmission type devices like smart windows and visors. 
Moreover, graphene has superior mechanical [15], optical [16], and electrical properties enabling its use in transparent electronic $[17,18]$ and photonic devices [19, 20]. These properties of graphene have made it a great candidate as a transparent conductive electrode. Graphene synthesized via chemical vapor deposition (CVD) on transition metals results in formation of large scale films with minimized structural defects, enabling its applications on an industrial scale [21]. In addition, studies have shown that controlled surface geometry of graphene can alter the wetting properties of the film, giving rise to superhydrophobic surfaces [22]. Here, we harness the mechanical instability of graphene to develop wrinkles on the film by applying radial strain to soft underlying substrate [23]. While PEDOT:PSS [24$26]$ and graphene $[27,28]$ have been used as conductive electrodes in electronics and photonics, the integrated electrochromic and hydrophobic properties of these materials have not been studied. We demonstrate a working electrochromic device which is flexible and stretchable, while exhibiting enhanced antiwetting properties. Graphene-based flexible electrochromic devices have been visualized in the past [29]. However, major challenges lie in developing a robust device structure which is free of liquid electrolytes that are hazardous to health and environment. Leakage of cell components is highly undesirable in optoelectronics, particularly in wearable devices. Here, we incorporate the electrolyte in a stretchable elastomer base which ensures an ionic conductivity and enables flexibility of the device, while enhancing safety.

Wrinkling or buckling in thin films is caused due to induced stresses in the components. The instability arises due to the compression of an interface leading to a deformation which propagates changes in surface properties. This outof-plane deformation of graphene enhances the adhesion energy of the stiff film to compliant substrates [30]. It is observed that the morphology of the generated wrinkles is largely dependent on the surface texture of the underlying substrate, with greater surface roughness contributing to higher wrinkling [31]. This reversible instability can be harnessed to generate diverse characteristics in surface morphologies that are of great importance in nanofabrication of elastic optoelectronic devices [32]. To this end, we employ a compliant elastomer (polyurethane) containing ionic liquid as dielectric medium and incorporate PEDOT:PSS/Xylitol films as the electrode, which exhibits electrochromic nature upon applying voltages, and graphene, which acts as the hydrophobic counterelectrode. Our device shows enhanced bleaching rate and stable functionality at variable strain rates envisioning its potential in flexible electronics. This novel application of stable, transparent, and mechanically flexible films provides a new perspective for optoelectronic devices.

\section{Experimental Procedure}

2.1. Preparation of Polyurethane Soft Substrate. Polyurethane (Clearflex $\left.{ }^{\mathrm{TM}}-50\right)$ was purchased from Smooth-On Inc. 1-Ethyl-3-methylimidazolium bis(trifluoromethylsulfonyl)imide(EMI-TFSI 99.97\%) ionic liquid, was procured from
Iolitec. The polyurethane (PU) gel was casted by mixing the isocyanate and polyol mixtures in a ratio of $1: 2$ by weight. EMI-TSFI (ionic liquid) was added to the elastomer mixture $20 \%$ by weight to impart the ionic conductivity to the inherent electrically insulating PU elastomer. The mixture was cast into polycarbonate petri dishes and degassed for 20 minutes. The mixture was allowed to dry at room temperature for 32 hours before peeling out of the dishes for device fabrication.

2.2. Preparing PDMS Substrate. Sylgard $-184^{\mathrm{TM}}$ Silicon elastomer (PDMS) was purchased from Dow corning. PDMS substrates were cast by mixing the elastomer base and the curing agent in a ratio of $10: 1$ by mass, followed by degassing. The mixture was cast in petri dishes and baked at $55^{\circ} \mathrm{C}$ for 3 hours.

2.3. Preparing PEDOT:PSS/Xylitol Films. Xylitol ( $\geq 99.99 \%$, crystalline PN: X3375) was purchased from Sigma-Aldrich. PEDOT:PSS (PH1000, 1-1.3 wt\% aqueous dispersion) was purchased from Ossila. Xylitol was added to the PEDOT:PSS aqueous dispersion in a ratio of $85 \%$ to the solid wt. of the solvent. In a typical experiment, $5 \mathrm{ml}$ solution of the PEDOT:PSS had $0.365 \mathrm{gm}$ of xylitol content. The solution mixture was flow-coated with a glass rod of $5 \mathrm{~mm}$ diameter onto the PDMS substrates which were sonicated with laboratory detergent for 20 minutes and cleaned with isopropanol and distilled water. The PDMS substrates were then treated with air plasma for 15 seconds prior to the casting in order to enhance the surface energy of PDMS and its wettability by PEDOT:PSS/Xylitol solution. Cast films were baked in an oven at $50^{\circ} \mathrm{C}$ for 5 hours for the water content to evaporate followed by heating at $120^{\circ} \mathrm{C}$ for 1 hour for the xylitol to melt and impart elasticity to the cast films.

2.4. Transfer of CVD Graphene. CVD graphene (6-8 layers) on copper was purchased from ACS material and ferric chloride based copper etchant (PN: 667528) was purchased from Sigma-Aldrich. Graphene was stamped to a $2 \mathrm{~mm}$ thick PDMS substrate and etched in the etching solution for 30 minutes (see supplementary materials, Figure S1). The graphene on PDMS was cleaned with deionized water thoroughly and blow-dried with air/nitrogen before transfer to the PU/IL substrate by stamping.

2.5. Device Fabrication. Cast $2 \mathrm{~mm}$ thick polyurethane/ionic liquid (PU/IL) substrate was cut in 2 in $\times 2$ in size and clamped between polycarbonate plates (see supplementary materials, Figure S2). The clamping enabled inducing radial strain by inflation of the substrate in the vertical plane. The PU/IL layer was inflated with compressed air controlling the flow rate for achieving desired prestrain conditions. Graphene on PDMS was stamped on the system in the inflated state, following which the substrate was deflated gradually. Cast PEDOT:PSS/Xylitol films were then transferred using a similar stamping process on the other side of the PU/IL, completing device fabrication analogous to a simple capacitor. Copper strips were used for making ohmic contact with the electrodes of the ECD. 


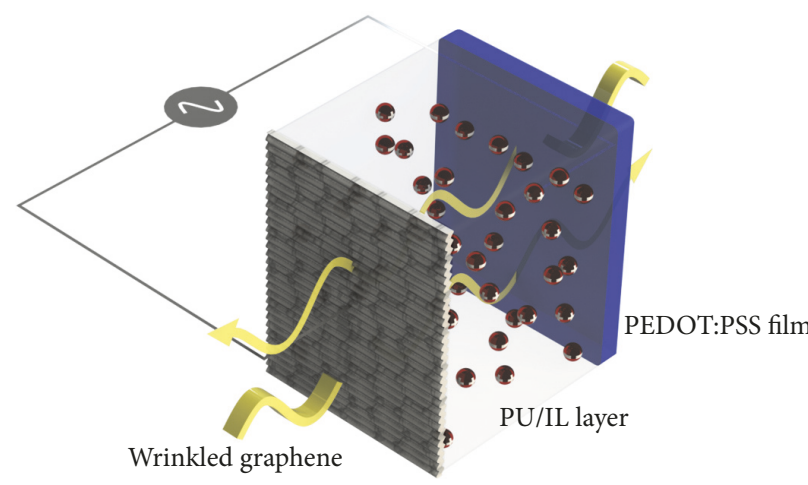

(a)

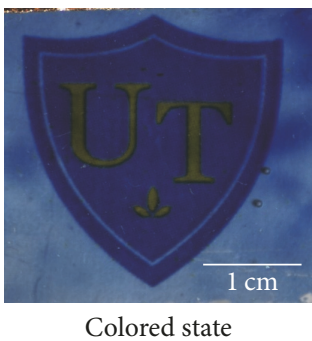

Colored state

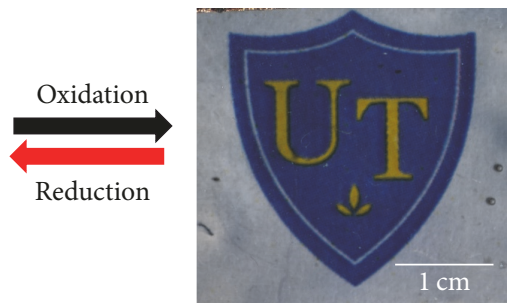

Bleached state

(b)

FIGURE 1: (a) Schematic of the electrochromic device (ECD) showing wrinkled graphene and PEDOT:PSS as transparent conductive electrodes and polyurethane/ionic liquid (PU/IL) as an elastic dielectric layer. (b) Photographs of the ECD at reduced (blue) state and oxidized (bleached) state under applied potential of $\pm 12 \mathrm{~V}$.

2.6. Characterization. Sheet resistances of the PEDOT:PSS/ Xylitol and graphene films were measured using a 4-point probe method. Recordings were taken from Keithly-2400 source meter. A minimum of 5 readings were taken across the film at various points to obtain an average value of the sheet resistance. Optical transmittance measurements were carried out using Perkin-Elmer-Lambda 1050 UV/VIS spectrometer. Scanning electron microcopy (SEM) images were obtained with a FEI-Quanta 3D-FEG dual beam electron microscope.

Static contact angle measurements were carried out by placing a water droplet of $3 \mu \mathrm{l}$ on the surface and capturing images with a DSLR camera (Nikon). The contact angles were measured using the image processing software ImageJ. A set of 5 readings were taken to obtain an average contact angle value.

The electrochemical properties of the device were measured using a Gamry 300 potentiostat/galvanostat. Cyclic voltammetry studies were carried out on PEDOT:PSS electrodes with a three-electrode cell design. $\mathrm{Ag} / \mathrm{Ag}^{+} \mathrm{Cl}^{-}$and platinum wires were used as the reference and counterelectrodes, while PEDOT:PSS/Xylitol was spin-coated on indium thin oxide (ITO) substrate as the working electrode. The electrolyte for the cell was prepared by adding $0.1 \mathrm{M}$ lithium perchlorate $\left(\mathrm{LiClO}_{4}\right)$ to dry acetonitrile.

\section{Results and Discussion}

3.1. Composition of the Electrochromic Device (ECD). Figure 1(a) shows a schematic structure of the electrochromic device. Polyurethane (PU) is a highly elastic and transparent polymer, with a transmissivity of $\sim 92 \%$ at $550 \mathrm{~nm}$ wavelength (see supplementary materials, Figure S3) and resistant to ultraviolet radiation, is an ideal choice for our soft and compliant substrate with elastic nature withstanding large strains. However, due to electrically nonconductive nature of the PU layer, an ionic liquid (IL) was added to the elastomer base [33]. Ionic liquids are salts which are highly stable at room temperature and have high ionic conductivity [34] which can withstand high thermal and chemical strains. A nonaqueous solvent (EMI-TFSI) was chosen as it was highly miscible with the PU [35] and had low vapor pressure and a wide electrochemical window [36], resulting in highly transparent and uniform PU gel layers with enhanced functionality. The ionic conductivity of the gel was measured to be in the range of $6 \times 10^{-5} \mathrm{~S} / \mathrm{cm}$, which is in agreement with previous reports [35].

It is highly crucial to develop and incorporate flexible conductors which can retain their electrical conductivity under high operating strains. Stretchable conductors have been used predominantly in organic photovoltaics (OPVs) and light emitting diodes (OLEDs) [37-39]. Wang et al. developed soft flexible PEDOT:PSS films which showed high conductivity over large strain rates [40]. Conductive polymers have allured research because of their tunable molecular structures that can control mechanical and electrical properties. Addition of plasticizers like sugar alcohols with more than two hydroxyl groups have also shown enhanced conductivity [41, 42] and elasticity [43] in PEDOT:PSS. The addition of a processing additive like xylitol helps in weakening the dominant hydrogen bonds between PSS and the PEDOT ionomers by negating their coulombic interactions in the dispersion medium [44]. The xylitol then forms secondary bonding (hydrogen bonding) with the PEDOT [35] which enhances flexibility and improves elastic nature after thermal annealing of the film near the melting point of the additive (xylitol melts at $94^{\circ} \mathrm{C}-97^{\circ} \mathrm{C}$ ), while imparting high bulk conductivity [45]. The resonant structure of the PEDOT changes from a benzenoid structure to a quinoid structure after adding a sugar alcohol [46] initiating an ordered planar backbone $[47,48]$. This invariably results in a higher packing order [49] causing a morphological change in the PEDOT:PSS/Xylitol film and forming a closer rearranged polymer interconnect hereby enhancing the conductivity. Typical sheet resistance of the cast PEDOT:PSS/Xylitol films with transparency of approximately $84 \%$ at $550 \mathrm{~nm}$ was measured to be $89 \pm 8 \Omega \cdot \mathrm{sq}^{-1}$. The figure of merit was calculated to be 23-31 using the following expression:

$$
\mathrm{FOM}=\frac{Z_{0}}{2 R_{s}\left(T^{-0.5}-1\right)},
$$


where $Z_{0}$ is the impedance of free space $(377 \Omega)$, T is the transmittance, and $R_{s}$ is the sheet resistance of the PEDOT:PSS film.

For the counterelectrode, graphene was a viable alternative layer because of its high electrical conductivity, extraordinary tensile strength, and transparency, envisioning its use in photonics and optoelectronics [50]. Optical properties and gate tunable characteristics of graphene have been an active research area in electronics [51-54]. Thin graphene films are transparent and enable an optical path changing their interference color with respect to the underlying substrate [55]. With absorbing around $2 \%$ of incident light in the visible spectrum [56], graphene acts as a superior electrode material for transparent electronics. PEDOT:PSS based electrochromic devices have primary and complementary electrochromes, where a simultaneous electrochemical reaction of the two redox-active layers takes place at applied charge. Our device, on the contrary, has a single active layer of electrochrome (PEDOT:PSS) which also works as a functioning transparent electrode. While graphene is reported to show a certain percolation effect on applied potentials, the authors report no significant change in transmittance for a multilayered (7-8 layers) graphene electrode. In addition, the transmittance values at $550 \mathrm{~nm}$ wavelength did not experience any considerable change due to induced wrinkles ( $T=$ $83.37 \%$ before and $T=83.26 \%$ after wrinkling). While wrinkling instability has previously been harnessed to prepare superhydrophobic surfaces, the wavelength so far has been limited to larger than microns, resulting in opaque surfaces $[57,58]$. By selection of compliant substrate and electrodes, we can control the critical strain $\left(\varepsilon_{c}\right)$ developed in the system, which regulates the wavelength of the wrinkles generated while maintaining transparency at the same time. Moreover, negligible light scattering even after enhancement of surface roughness by wrinkling of few layers of graphene enables its application as a transparent conductor. The mechanics of the wrinkling is discussed in detail later.

3.2. Electrochromic Response of the Device. The performance of the device was investigated by testing the assembled components in a hierarchical sandwich model with the wrinkled graphene and PEDOT:PSS/Xylitol films as the reversible electrodes and the PU/IL gel acting as the solid state electrolytic medium. The net transparency of the device was measured to be $\sim 70 \%$ while the transparency of PEDOT:PSS/Xylitol film was $84.58 \%$ (see supporting information, Figure S3). When the two conductive films experience a substantial potential difference, the solid state electrolyte develops an electric field which propagates an accumulation of charges at the conductor plates. This flow of charge across the electrolyte triggers an electrochemical redox reaction in the PEDOT:PSS layers enabling its electrochromic behavior and leading to the color-switching phenomenon, which can be explained by the following reaction:

$$
\mathrm{PEDOT}^{+} \mathrm{PSS}^{-}+M^{+}+e^{-} \longleftrightarrow \mathrm{PEDOT}^{0}+\mathrm{PSS}^{-} M^{+} .
$$

Figure 1(b) shows the transition between the reduced and oxidized states of the device during an induced charge of $12 \pm 0.1 \mathrm{~V}$. The polarity of the electrodes was changed at a pulse width of 40 seconds. PEDOT:PSS undergoes reduction reaction when a negative voltage bias is applied while the absorption over the visual light range (1.6-3.4 eV) is greatly enhanced. On the contrary, a positive voltage bias causes oxidation leading to minimal absorption in the visible light range. This change in absorption of light enables the monochromatic color switch contrast between darker and lighter shades of blue. Figure 2(a) shows the transmittance values of the device in the reduced and oxidized states. The transparency at the peak oxidation and reduction states at $550 \mathrm{~nm}$ was observed to be $70.36 \%$ and $45.2 \%$, respectively. The contrast ratio $(\Delta \% T)$ is calculated to be $25.16 \%$. The transmittance curves vary between duplicates of the device assembled (not shown) with variable transparency values of the PEDOT:PSS/Xylitol films. A PEDOT:PSS/Xylitol film with a transparency of $\sim 73 \%$ (thickness $\sim 500 \mathrm{~nm}$ ) resulted in a device transparency of $\sim 61 \%$, and it is observed that the transparency decreases further with thicker PEDOT:PSS/xylitol films. This suggests that the overall transparency change of the devices was dependent upon the transmittance of the cast PEDOT:PSS films. The thicker films having lesser transmittance showed higher color contrast between the oxidized and the reduced states for the same induced charge, while the transparency of the other bilayers, namely, graphene and PU/IL, was kept homogeneous across all fabricated devices (see supplementary materials, Figure S3).

To further investigate the dynamic response of our device, a series of time dependent tests were performed with variable voltages and the transmittance was observed at a wavelength of $550 \mathrm{~nm}$. Figure 2(b) shows transmittance as a function of time with variable applied voltage for a period of 200 seconds. The electrochromic response of the device remains constant with time. It is also observed that the time taken to reach the peak oxidation and reduction states is more for lower potentials, resulting in higher response times. The electrical response of the device can be improved by selective postdeposition chemical treatment of graphene, to enhance its conductivity and charge carrier concentration $[59,60]$. A considerable impedance is also observed in the PU gel interlayer resulting in a higher voltage to drive the device. While the experiments were performed with a $20 \%$ ionic liquid composition in the PU matrix, the authors believe that a greater efficiency in terms of lower driving potentials can be achieved by decreasing the effective thickness of the electrolyte $(<1 \mathrm{~mm})$ with simultaneous increase of ionic content in the PU gel matrix. The cyclic voltammetry plot for PEDOT:PSS with 85\% xylitol additive is shown in Figure 2(c). The current versus voltage plots were generated at a scan rate of $80 \mathrm{mv} / \mathrm{s}$ between a potential window of $-1.2 \mathrm{~V}$ to $0.8 \mathrm{~V}$. The electrochromes were spin-coated on ITO substrate, which acts as our functional electrode. Cyclic voltammetry evaluates the redox response of the electrochrome which depends on the conductivity of the film. It is seen that the oxidation and the reduction peaks are identical indicating greater reversibility of the redox couple. A wider window for the PEDOT:PSS/xylitol film ascertains an enhanced electrochemical behavior, higher charge capacity, and electron 


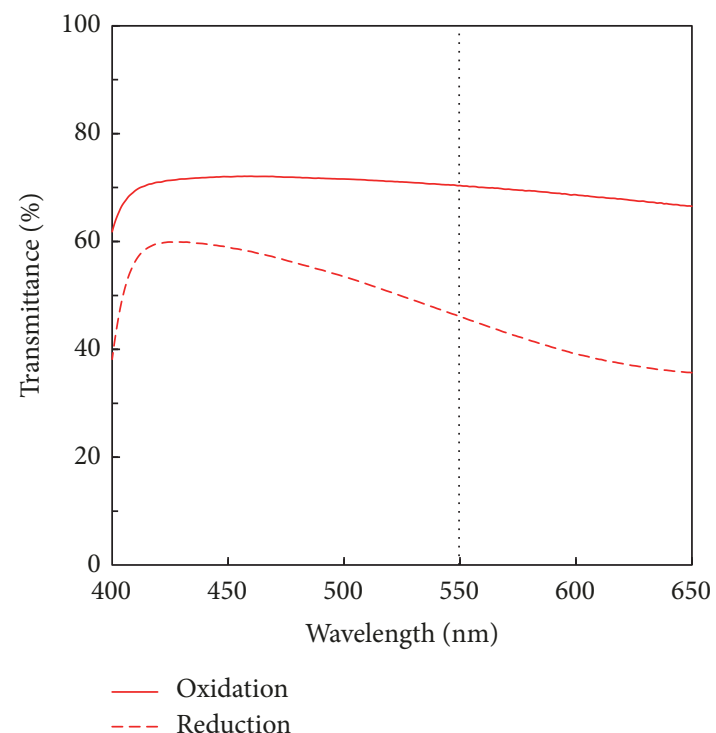

(a)

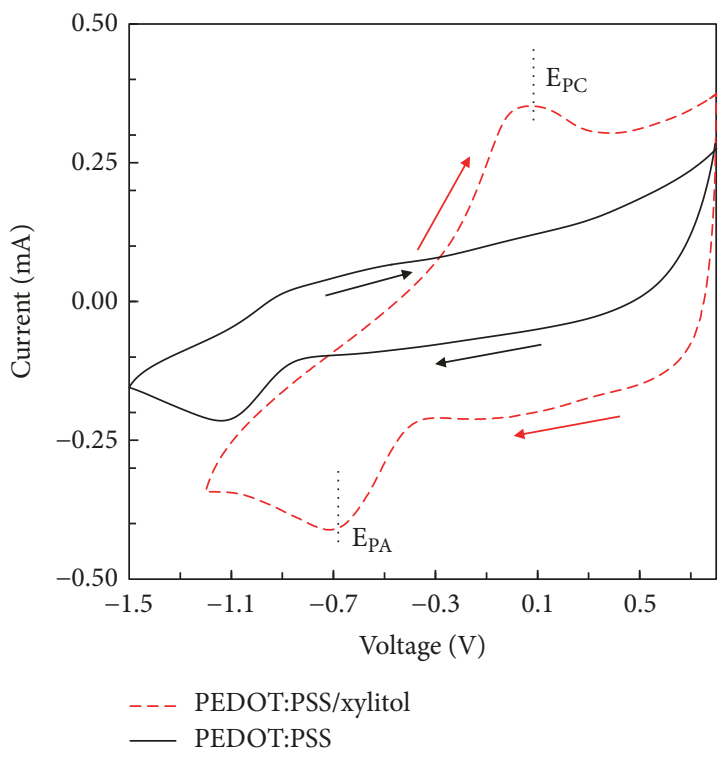

(c)

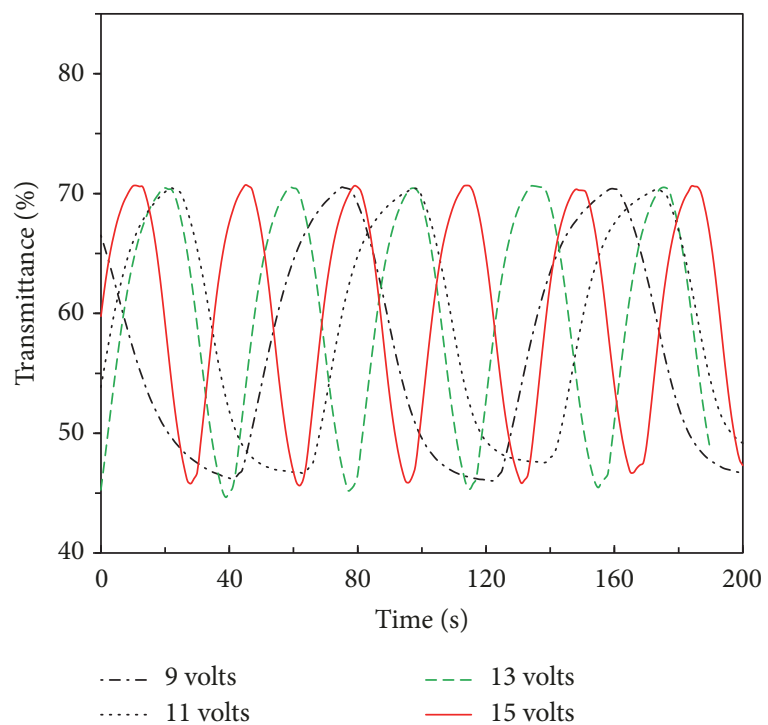

(b)

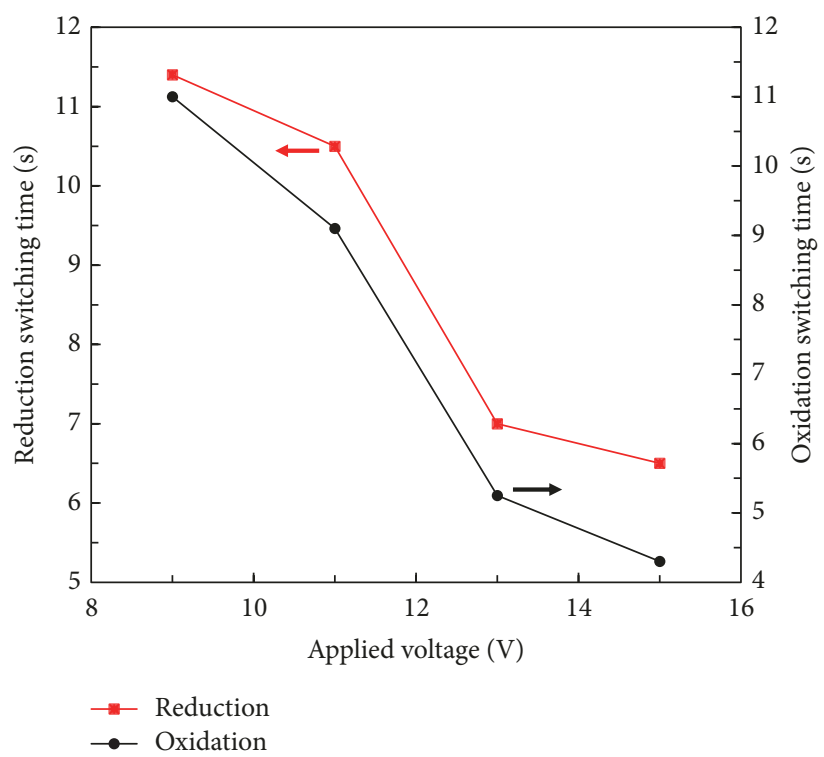

(d)

FIGURE 2: (a) Oxidation and reduction response plot for the electrochromic device (ECD). The transmittance (\%) at $550 \mathrm{~nm}$ wavelength for reduced and oxidized states is $70.36 \%$ and $45.2 \%$, respectively. (b) Time versus transmittance response curves for the device under variable applied voltages. The transmittance values were recorded for a period of 200 seconds with reversing polarity of the electrodes. (c) Cyclic voltammetry plot for PEDOT:PSS/xylitol and PEDOT:PSS. The coloration efficiency was calculated to be in the order of $80 \mathrm{~cm}^{2} \mathrm{C}^{-1}$. (d) Plot for switching time in oxidation and reduction states of the ECD (switching times were calculated for $90 \%$ change in transmittance $(66.84 \%-47.57 \%)$ of the device with respect to the equilibrium value).

mobility increase in the films. The coloration efficiency of the films was calculated to be $80.49 \mathrm{~cm}^{2} \mathrm{C}^{-1}$ for a $95 \%$ change in transmission at $550 \mathrm{~nm}$ wavelength. The electrochemical characterization of the CVD-grown multilayer graphene films has been performed elsewhere, the kinetics of which depend on the surface geometry and quality of the graphene films [61]. Figure 2(d) shows the switching speeds of the ECD as a function of applied voltage for a $90 \%$ change in transmittance (with respect to equilibrium value, $67.57 \%$ to $47.57 \%)$ at $550 \mathrm{~nm}$ wavelength. The decrease in response time at higher potential differences can be attributed to the greater mobility of charge carriers and faster redox kinetics of the PEDOT:PSS electrode.

To test for the robustness of the ECD, a set of experiments was performed on another device with a net transparency of $\sim 61 \%$. Figure 3 (a) qualitatively shows the functionality and the variation in transmittance while the device was flexed. The device was then stretched uniaxially at strain rates of $\varepsilon=0.10$ and $\varepsilon=0.15$ and the transmittance tabulated accordingly (see Table 1). Figure 3(b) shows the 


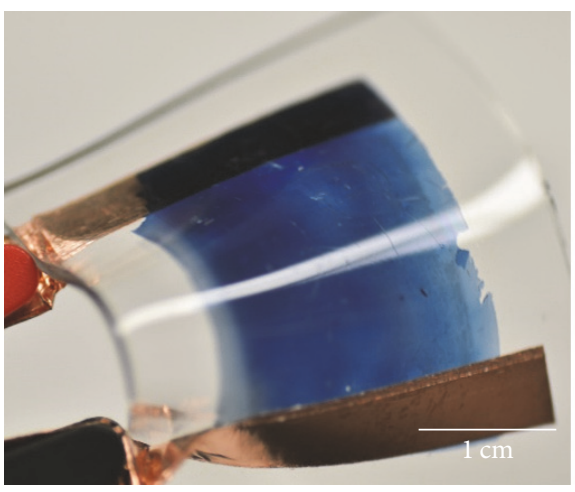

Colored state

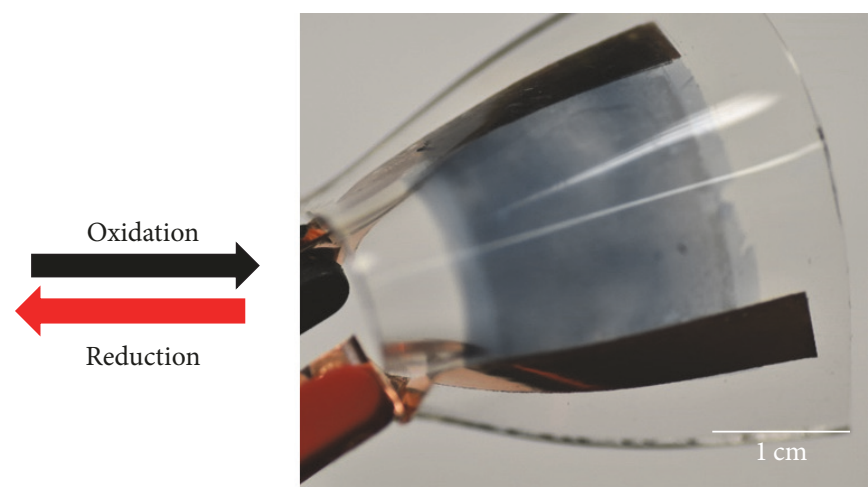

Bleached state

(a)

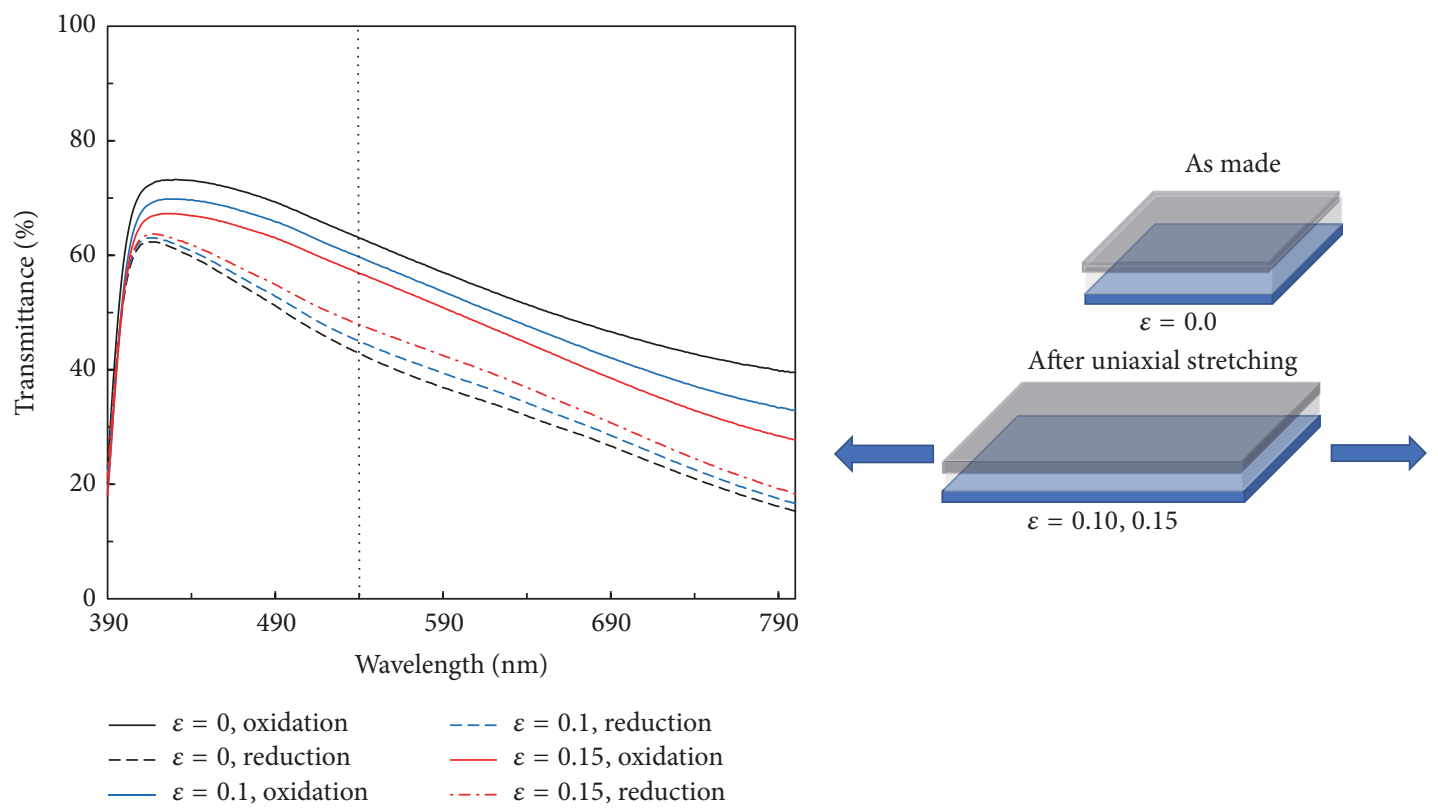

(b)

FIGURE 3: (a) Photographs of the flexible electrochromic device (ECD) in reduced (blue) and oxidized (bleached) states while it is being flexed under applied voltage of $\pm 15 \mathrm{~V}$, showing a steady color change. (b) Change in transmittance percentage with variable uniaxial strains of $\varepsilon=0$, $\varepsilon=0.1$, and $\varepsilon=0.15$. The reduction transparency of the device increases with applied strains with a net transparency of $41.48 \%$ at $\varepsilon=0.0$ to $46.81 \%$ at $\varepsilon=0.15$. A simultaneous decrease in transmittance is observed at the oxidized state with $61.84 \%$ transmittance at $\varepsilon=0.0$ to $55.72 \%$ transmittance at $\varepsilon=0.15$. The schematic (right) depicts the method of applied strain on the device.

TABLE 1: Variation in oxidized (bleached) and reduced (blue) transmittance (\%) with applied strains. The device was stretched uniaxially (as shown in Figure 3(b)). The transparency of the device varied by $9.89 \%$ in the oxidized state and $12.84 \%$ in the reduced states for strain rate of $\varepsilon=0.0$ to $\varepsilon=0.15$.

\begin{tabular}{lcc}
\hline \multirow{2}{*}{ Strain rate $(\varepsilon)$} & \multicolumn{2}{c}{ Transmittance (\%) } \\
& Oxidized & Reduced \\
\hline 0.0 & 61.84 & 41.48 \\
0.10 & 58.44 & 43.77 \\
0.15 & 55.72 & 46.81 \\
\hline
\end{tabular}

transmittance responses in oxidation and reduction states at different strain rates. It is observed that the transmittance values in the oxidized state vary by $9.89 \%$ at $\varepsilon=0$ to $\varepsilon=0.15$ strain rates while the transmittance in reduced state increased by $12.84 \%$. There is a considerable change in the transmittance $(\Delta T)$ of the device after being stretched, which can be addressed by a more robust deposition method for the electrochrome to retain lower $\Delta T$ values. It is to be noted that the device retained its electrochromic nature even at $\varepsilon=0.15$ strain rate. The device was kept at the extended (stretched) state for 240 seconds before the electrochromic response was measured consecutively for all measured strain rates. The stability studies (for extended time) of the PEDOT:PSS thin film were not carried out in this study to arrive at a conclusion in terms of durability. However, the consecutive runs in pulses of 15 minutes showed a robust and a dynamic response without any drastic change 
in the optical properties of the device. It is also observed that the applied strain beyond which the device fails is limited to the maximum normal strain that the graphene film can withstand, which is $20 \%$ prior to fracture [62]. Change in transparency of the device can be due to the density variations in the crystal structure upon stretching which invariably cause differential light scattering and reflection. The additive chains tend to be localized in the more disordered channels of the PEDOT:PSS interconnect contributing to a softer material matrix [40]. This matrix can realign upon stretching resulting in an increase in transparency without affecting the poly-ion bridges and the overall conductivity of the film. The fractional increase and decrease in transparency of the device during intermittent stretching may be due to a greater surface area of the films induced by stretching; this decreases the effective concentration per unit area of the ions impacting the electrochromic response.

3.3. Wrinkling of Graphene. Introducing wrinkled graphene as the counterelectrode enhanced the functionality of our device as it exhibits antiwetting properties [22]. Nonlinear buckling in compliant substrates can be explained by the energy balance theory [63]. The total energy at work on the systems for an equilibrium state is the elastic energy of the soft substrate (in this case, PU/IL) and the bending energy of the underlying stiff films with a larger Young's modulus. The adhesion energies of graphene and the substrate and the graphene deformation define the equilibrium state at which graphene conforms with the elastic substrate. In consideration, we assume that the elastic substrate is incompressible and, due to its soft nature, the elastic energy contributes to the net equilibrium energy, hereby defining the adhesion energy of the PU. The wavelengths generated by this mechanical instability of graphene tend to adhere to an equilibrium state where these bending and stretching forces are minimized. In theory, a reduced wavelength inclines more towards a minimized stretching energy of the elastic substrate whereas a higher wavelength buckling tends to initiate deformations at the expense of lower bending energies of the stiff film. The ideal wavelength of the system is governed by the thickness of the stiff film and the modulus contrast in the material properties.

Graphene is superelastic and stiff as the carbon atoms in graphene can be flexed relatively easily without breaking the strong covalent bonds between the atoms. The critical strain $\left(\varepsilon_{c}\right)$ at which the buckling occurs is given as a contrast between the plain strain moduli of the stiff and flexible films [23]:

$$
\varepsilon_{c}=\frac{1}{4}\left(\frac{3 \bar{E}_{s}}{\bar{E}_{f}}\right)^{2 / 3}
$$

where $\bar{E}_{f}$ and $\bar{E}_{s}$ are the plain strain moduli of the film and the soft substrate, given by $\bar{E}=E /\left(1-v^{2}\right) ; E$ and $v$ are Young's modulus and the Poisson's ratio of the film and the soft substrate. The buckling wavelength and amplitude can be calculated by the energy balance theory and can be explained by 2nd-order Piola-Kirchhoff's stress formulation for neoHookean materials. The prestrain dependent wavelength and amplitude can be expressed as [23]

$$
\begin{aligned}
& \lambda=\frac{\lambda_{0}}{\left(1+\varepsilon_{\text {pre }}\right)(1+\xi)^{1 / 3}}, \\
& A=\frac{A_{0}}{\sqrt{\left(1+\varepsilon_{\text {pre }}\right)}(1+\xi)^{1 / 3}},
\end{aligned}
$$

where $\lambda_{0}=2 \pi h\left(3 \bar{E}_{f} / \bar{E}_{s}\right)^{1 / 3}$ and $A_{0}=h \sqrt{\varepsilon_{\text {pre }} / \varepsilon_{c}-1}$ are the wavelength and amplitude of the film for lower strain rates $\left(\varepsilon_{\text {pre }}<5 \%\right) . \xi=(5 / 32) \varepsilon_{\text {pre }}\left(1+\varepsilon_{\text {pre }}\right)$ is a function of the applied prestrain and $(1+\xi)^{1 / 3}$ is obtained due to the nonlinear geometry caused by large deformation of the substrate. Assuming Young's modulus and Poisson's ratio of graphene as $1 \mathrm{Tpa}$ and 0.165 , and of polyurethane (PU) as $2.5 \mathrm{Mpa}$ and 0.5 , we obtain a wavelength $\lambda_{0}=2.293 \mu \mathrm{m}$. The thickness of the CVD-grown ML graphene film was assumed to be $10 \mathrm{~nm}$ [64]. The resultant height of the wrinkles generated was theoretically calculated using (4). The theoretical amplitude was in the order of $453.06 \mathrm{~nm}$ which contributed to change in surface characteristics of the graphene film resulting in its antiwetting nature.

Figure 4(a) shows the water contact angles of polyurethane substrate, pristine multilayered graphene, and wrinkled graphene (induced prestrain $=0.3$ ). The graphene films were transferred to the PU substrate before measuring the contact angles. A contact angle of $110^{\circ} \pm 2^{\circ}$ was measured for wrinkled graphene samples indicating an increase in antiwetting nature of the graphene film. The contact angle measurements for bare polyurethane substrate and pristine graphene were $59.47^{\circ} \pm 3^{\circ}$ and $91.96 \pm 3^{\circ}$, respectively. Figure 4(b) shows the hydrophobic nature of the device with color contrast in the reduced and oxidized states (see supplementary materials, S4). The wavelength was verified with SEM imaging (Figure 4(c)) and was found close to our calculations. It is observed that the contact angles increase with higher prestrains with reported contact angles as high as $152^{\circ}$ at applied prestrains of $400 \%$ [22].

The surface morphology of graphene can be further tuned to exhibit higher water contact angle values while exhibiting superior conductivity. This along with a use of an organic polymer like PEDOT:PSS as a flexible transparent conductive electrode can result in developing ECDs with enhanced functionalities, enabling their application in areas where there is frequent exposure to water stretching/flexing conditions. Devices with water repelling electrodes like graphene can be envisioned in outdoor applications where there is frequent exposure to atmospheric moisture and water. Also, the device can be further enhanced by incorporating ultrathin conformal polymeric coatings which can prevent the active electrochrome as well, hereby enabling a complete waterproof ECD. This study explores avenues for incorporating wrinkled graphene to develop robust PEDOT:PSS based electrochromic devices with antiwetting properties and establishes groundwork for developing flexible, robust, 


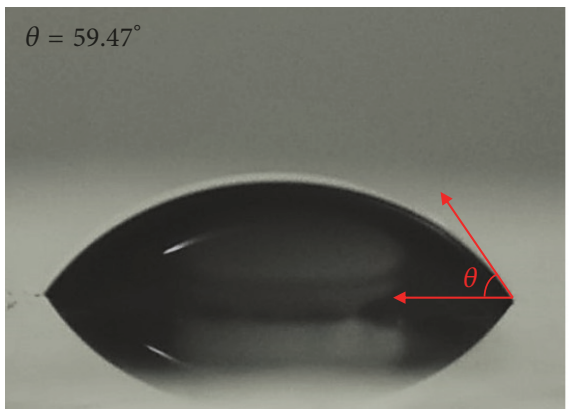

Bare substrate

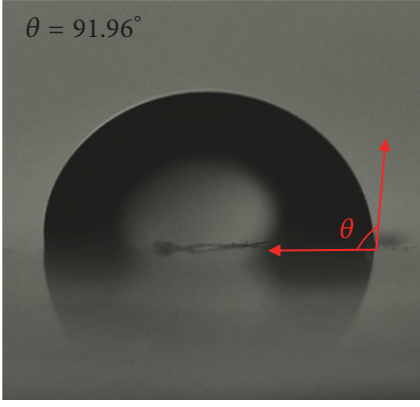

Pristine graphene

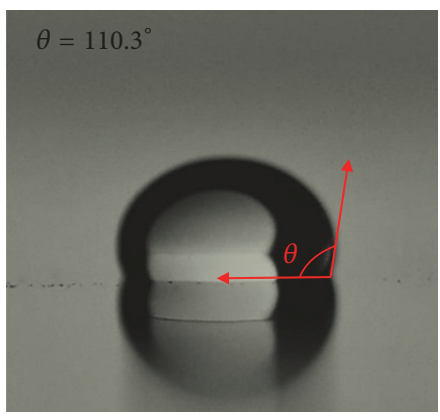

Wrinkled graphene

(a)

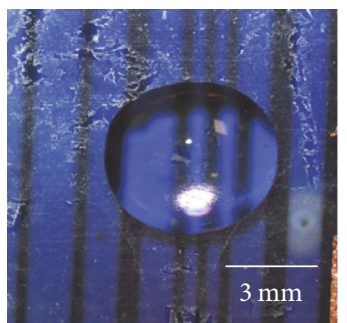

Colored state

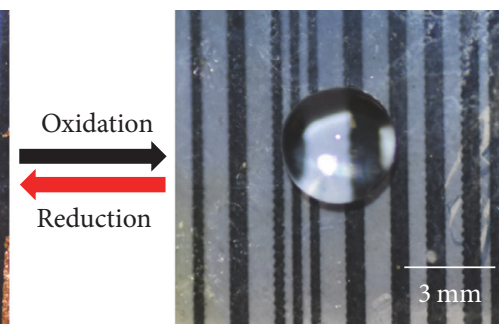

Bleached state

(b)

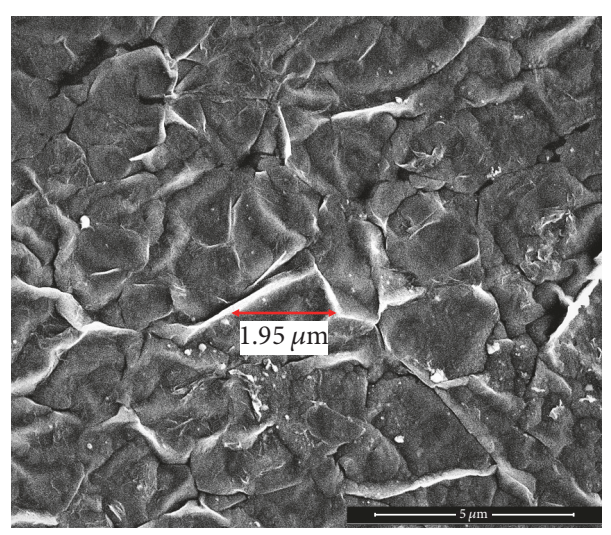

(c)

FIGURE 4: (a) Water contact angle measurements on polyurethane, pristine graphene, and wrinkled graphene. Wrinkling results in controlled formation of roughness in graphene and subsequent increase in its water contact angle values. (b) Hydrophobic nature of the electrochromic device, shown by a water droplet placed on the device, exhibiting a contact angle of $110^{\circ} \pm 2^{\circ}$ in oxidized (bleached) and reduced (blue) states. (c) SEM image of the wrinkled graphene at an applied radial prestrain of $30 \%$. The wrinkles have a periodicity of $\sim 1.95 \mu \mathrm{m}$, which is on the same order of magnitude with our calculated wavelength of $2.293 \mu \mathrm{m}$.

and durable low-cost optoelectronic gadgets with improved efficiencies and wider functionalities.

\section{Conclusion}

In summary, we fabricated a flexible and transparent electrochromic device with graphene and PEDOT:PSS with xylitol as a processing additive as the interchangeable electrodes while PEDOT:PSS functions as an electrochromic material. The ionic medium in this parallel plate capacitor geometry was incorporated in the transparent and elastic polyurethane substrate enabling a nonhazardous and an environmentally friendly device structure. We also successfully demonstrated color switching of the device at variable strain rates confirming its potential for application in flexible and stretchable electronics. The switching times for the device during the oxidation and reduction states and their impact on optical characteristics were studied. The device also exhibits antiwetting properties through modifying the morphology of the graphene film via inducing wrinkling effect by applied prestrains. Therefore, the properties of PEDOT:PSS and graphene can both be envisioned together in developing ECDs with better functionality and efficient performance.

\section{Conflicts of Interest}

The authors declare that there are no conflicts of interest regarding the publication of this article.

\section{Supplementary Materials}

Figure S1: schematic diagram of the graphene film transfer from the copper substrate to PDMS using stamping technique. Figure S2: setup for inflating the polyurethane/ionic liquid (PU/IL) layer by applying radial prestrain via air supply. Graphene on PDMS was stamped on the inflated PU/IL; controlled wrinkles were obtained in graphene upon gradual deflation of the PU/IL layer. Figure S3: transmittance plots for multilayer (6-8 layers) graphene, PEDOT:PSS/Xylitol, and polyurethane/IL gel. Figure S4: additional images of water droplet $(3-4 \mu \mathrm{l})$ on the electrochromic device (ECD) in oxidized (bleached) and reduced (blue) states, exhibiting hydrophobic nature. (Supplementary Materials)

\section{References}

[1] J. Jensen, M. Hösel, A. L. Dyer, and F. C. Krebs, "Development and manufacture of polymer-based electrochromic devices," 
Advanced Functional Materials, vol. 25, no. 14, pp. 2073-2090, 2015.

[2] P. N. Moskalev and I. S. Kirin, "ChemInform Abstract: synth. und einige eigenschaften von yttrium- und erbiumdiphthalocyanin," Optics and Spectroscopy, vol. 29, no. 1970, pp. no-414, 1970.

[3] Y.-H. Tak, K.-B. Kim, H.-G. Park, K.-H. Lee, and J.-L. Lee, "Criteria for ITO (indium-tin-oxide) thin film as the bottom electrode of an organic light emitting diode," Thin Solid Films, vol. 411, pp. 12-16, 2002.

[4] Y. Shen, D. B. Jacobs, G. G. Malliaras, G. Koley, M. G. Spencer, and A. Ioannidis, "Modification of indium tin oxide for improved hole injection in organic light emitting diodes," Advanced Materials, vol. 13, no. 16, pp. 1234-1238, 2001.

[5] J. F. Wager, “Transparent electronics," Science, vol. 300, no. 5623, pp. 1245-1246, 2003.

[6] C. G. Granqvist, "Transparent conductors as solar energy materials: a panoramic review," Solar Energy Materials \& Solar Cells, vol. 91, no. 17, pp. 1529-1598, 2007.

[7] P. Monk, R. Mortimer, and D. Rosseinsky, Electrochromism fundamentals and applications, VCH Publishers, Inc., New York, NY, USA, 1995.

[8] R. J. Mortimer, "Organic electrochromic materials," Electrochimica Acta, vol. 44, no. 18, pp. 2971-2981, 1999.

[9] R. J. Mortimer, A. L. Dyer, and J. R. Reynolds, "Electrochromic organic and polymeric materials for display applications," Displays, vol. 27, no. 1, pp. 2-18, 2006.

[10] A. A. Argun, P.-H. Aubert, B. C. Thompson et al., "Multicolored electrochromisin in polymers: Structures and devices," Chemistry of Materials, vol. 16, no. 23, pp. 4401-4412, 2004.

[11] A. A. Argun, A. Cirpan, and J. R. Reynolds, "The First Truly AllPolymer Electrochromic Devices," Advanced Materials, vol. 15, no. 16, pp. 1338-1341, 2003.

[12] L. Groenendaal, F. Jonas, D. Freitag, H. Pielartzik, and J. R. Reynolds, "Poly(3,4-ethylenedioxythiophene) and its derivatives: past, present, and future," Advanced Materials, vol. 12, no. 7, pp. 481-494, 2000.

[13] H. Yan, T. Jo, and H. Okuzaki, "Potential application of highly conductive and transparent poly(3,4-ethylenedioxythiophene)/ poly(4-styrenesulfonate) thin films to touch screen as a replacement for indium tin oxide electrode," Polymer Journal, vol. 43, no. 7, pp. 662-665, 2011.

[14] Y. H. Kim, C. Sachse, M. L. MacHala, C. May, L. MüllerMeskamp, and K. Leo, "Highly conductive PEDOT:PSS electrode with optimized solvent and thermal post-treatment for ITO-free organic solar cells," Advanced Functional Materials, vol. 21, no. 6, pp. 1076-1081, 2011.

[15] A. K. Geim and K. S. Novoselov, "The rise of graphene," Nature Materials, vol. 6, no. 3, pp. 183-191, 2007.

[16] R. R. Nair, P. Blake, A. N. Grigorenko et al., "Fine structure constant defines visual transparency of graphene," Science, vol. 320, no. 5881, p. 1308, 2008.

[17] H. Sojoudi and S. Graham, "Transfer-free selective area synthesis of graphene using solid-state self-segregation of carbon in $\mathrm{Cu} / \mathrm{Ni}$ Bilayers," ECS Journal of Solid State Science and Technology, vol. 2, no. 6, pp. M17-M21, 2013.

[18] J. Baltazar, H. Sojoudi, S. A. Paniagua et al., "Facile formation of graphene P-N junctions using self-assembled monolayers," The Journal of Physical Chemistry C, vol. 116, no. 36, pp. 19095-19103, 2012.
[19] H. Sojoudi, J. Baltazar, L. M. Tolbert, C. L. Henderson, and S. Graham, "Creating graphene $p-n$ junctions using selfassembled monolayers," ACS Applied Materials \& Interfaces, vol. 4, no. 9, pp. 4781-4786, 2012.

[20] H. Sojoudi, J. Baltazar, L. Tolbert, C. Henderson, and S. Graham, "Formation of air stable graphene p-n-p junctions using an amine-containing polymer coating," Advanced Materials Interfaces, vol. 1, no. 9, Article ID 1400378, 2014.

[21] X. Li, W. Cai, J. An et al., "Large-area synthesis of high-quality and uniform graphene films on copper foils," Science, vol. 324, no. 5932, pp. 1312-1314, 2009.

[22] J. Zang, S. Ryu, N. Pugno et al., "Multifunctionality and control of the crumpling and unfolding of large-area graphene," Nature Materials, vol. 12, no. 4, pp. 321-325, 2013.

[23] D.-Y. Khang, J. A. Rogers, and H. H. Lee, "Mechanical buckling: Mechanics, metrology, and stretchable electronics," Advanced Functional Materials, vol. 19, no. 10, pp. 1526-1536, 2009.

[24] R. Singh, J. Tharion, S. Murugan, and A. Kumar, "ITOFree Solution-Processed Flexible Electrochromic Devices Based on PEDOT:PSS as Transparent Conducting Electrode," ACS Applied Materials \& Interfaces, vol. 9, no. 23, pp. 19427-19435, 2017.

[25] X. Zhang, J. Wu, J. Wang et al., "Highly conductive PEDOT:PSS transparent electrode prepared by a post-spin-rinsing method for efficient ITO-free polymer solar cells," Solar Energy Materials \& Solar Cells, vol. 144, pp. 143-149, 2016.

[26] Z. Li, Y. Liang, Z. Zhong et al., "A low-work-function, highconductivity PEDOT:PSS electrode for organic solar cells with a simple structure," Synthetic Metals, vol. 210, pp. 363-366, 2015.

[27] Y. Xu and J. Liu, "Graphene as Transparent Electrodes: Fabrication and New Emerging Applications," Small, vol. 12, no. 11, pp. 1400-1419, 2016.

[28] H. Sojoudi, F. Reiter, and S. Graham, "Transparent Electrodes From Graphene/Single Wall Carbon Nanotube Composites," in Proceedings of the ASME 2013 International Technical Conference and Exhibition on Packaging and Integration of Electronic and Photonic Microsystems, p. V001T07A008, Burlingame, California, USA.

[29] E. O. Polat, O. Balc, and C. Kocabas, "Graphene based flexible electrochromic devices," Scientific Reports, vol. 4, article 6484, 2014.

[30] S. P. Koenig, N. G. Boddeti, M. L. Dunn, and J. S. Bunch, "Ultrastrong adhesion of graphene membranes," Nature Nanotechnology, vol. 6, no. 9, pp. 543-546, 2011.

[31] S. Deng and V. Berry, "Wrinkled, rippled and crumpled graphene: An overview of formation mechanism, electronic properties, and applications," Materials Today, vol. 19, no. 4, pp. 197-212, 2016.

[32] D. Chen, J. Yoon, D. Chandra, A. J. Crosby, and R. C. Hayward, "Stimuli-responsive buckling mechanics of polymer films," Journal of Polymer Science Part B: Polymer Physics, vol. 52, no. 22, pp. 1441-1461, 2014.

[33] T. Iwata, A. Tsurumaki, S. Tajima, and H. Ohno, "Bis(trifluoromethanesulfonyl)imide-type ionic liquids as excellent antistatic agents for polyurethanes," Macromolecular Materials and Engineering, vol. 299, no. 7, pp. 794-798, 2014.

[34] X. Fan and L. Wang, "Highly conductive ionic liquids toward high-performance space-lubricating greases," ACS Applied Materials \& Interfaces, vol. 6, no. 16, pp. 14660-14671, 2014.

[35] Y. Li, R. Tanigawa, and H. Okuzaki, "Soft and flexible PEDOT/PSS films for applications to soft actuators," Smart Materials and Structures, vol. 23, no. 7, Article ID 074010, 2014. 
[36] M. P. S. Mousavi, B. E. Wilson, S. Kashefolgheta et al., "Ionic Liquids as Electrolytes for Electrochemical Double-Layer Capacitors: Structures that Optimize Specific Energy," ACS Applied Materials \& Interfaces, vol. 8, no. 5, pp. 3396-3406, 2016.

[37] S. Wagner and S. Bauer, "Materials for stretchable electronics," MRS Bulletin, vol. 37, no. 3, pp. 207-213, 2012.

[38] I. Jung, J. Xiao, V. Malyarchuk et al., "Dynamically tunable hemispherical electronic eye camera system with adjustable zoom capability," Proceedings of the National Acadamy of Sciences of the United States of America, vol. 108, no. 5, pp. 17881793, 2011.

[39] X. Hu, P. Krull, B. De Graff, K. Dowling, J. A. Rogers, and W. J. Arora, "Stretchable inorganic-semiconductor electronic systems," Advanced Materials, vol. 23, no. 26, pp. 2933-2936, 2011.

[40] Y. Wang, C. Zhu, R. Pfattner et al., "A highly stretchable, transparent, and conductive polymer," Science Advances, vol. 3, no. 3, p. e1602076, 2017.

[41] E. Yang, J. Kim, B. J. Jung, and J. Kwak, "Enhanced thermoelectric properties of sorbitol-mixed PEDOT:PSS thin films by chemical reduction," Journal of Materials Science: Materials in Electronics, vol. 26, no. 5, pp. 2838-2843, 2015.

[42] A. M. Nardes, M. Kemerink, M. M. de Kok, E. Vinken, K. Maturova, and R. A. J. Janssen, "Conductivity, work function, and environmental stability of PEDOT:PSS thin films treated with sorbitol," Organic Electronics, vol. 9, no. 5, pp. 727-734, 2008.

[43] Y. Li, Y. Masuda, Y. Iriyama, and H. Okuzaki, "Stretchable and Highly Conductive Polymer Films," Transactions of the Materials Research Society of Japan, vol. 37, no. 2, pp. 303-306, 2012.

[44] A. Elschner, PEDOT : principles and applications of an intrinsically conductive polymer, CRC Press, 1st edition, 2011.

[45] S. Timpanaro, M. Kemerink, F. J. Touwslager, M. M. De Kok, and S. Schrader, "Morphology and conductivity of PEDOT/PSS films studied by scanning-tunneling microscopy," Chemical Physics Letters, vol. 394, no. 4-6, pp. 339-343, 2004.

[46] P. Sze, K. Lee, P. Huang, D. Chou, B. Kao, and C. Huang, "The Investigation of High Quality PEDOT:PSS Film by MultilayerProcessing and Acid Treatment," Energies, vol. 10, no. 12, p. 716, 2017.

[47] S. Garreau, G. Louarn, J. P. Buisson, G. Froyer, and S. Lefrant, "In situ spectroelectrochemical Raman studies of poly $(3,4-$ ethylenedioxythiophene) (PEDT)," Macromolecules, vol. 32, no. 20, pp. 6807-6812, 1999.

[48] J. Ouyang, “"secondary doping” methods to significantly enhance the conductivity of PEDOT:PSS for its application as transparent electrode of optoelectronic devices," Displays, vol. 34, no. 5, pp. 423-436, 2013.

[49] J. Ouyang, C.-W. Chu, F.-C. Chen, Q. Xu, and Y. Yang, "Highconductivity poly(3,4-ethylenedioxythiophene):poly(styrene sulfonate) film and its application in polymer optoelectronic devices," Advanced Functional Materials, vol. 15, no. 2, pp. 203-208, 2005.

[50] F. Bonaccorso, Z. Sun, T. Hasan, and A. C. Ferrari, "Graphene photonics and optoelectronics," Nature Photonics, vol. 4, no. 9, pp. 611-622, 2010.

[51] A. Majumdar, J. Kim, J. Vuckovic, and F. Wang, "Electrical control of silicon photonic crystal cavity by graphene," Nano Letters, vol. 13, no. 2, pp. 515-518, 2013.
[52] X. Gan, R.-J. Shiue, Y. Gao et al., "Chip-integrated ultrafast graphene photodetector with high responsivity," Nature Photonics, vol. 7, no. 11, pp. 883-887, 2013.

[53] T. Mueller, F. Xia, and P. Avouris, "Graphene photodetectors for high-speed optical communications," Nature Photonics, vol. 4, no. 5, pp. 297-301, 2010.

[54] E. O. Polat and C. Kocabas, "Broadband optical modulators based on graphene supercapacitors," Nano Letters, vol. 13, no. 12, pp. 5851-5857, 2013.

[55] P. Blake, E. W. Hill, A. H. Castro Neto et al., "Making graphene visible," Applied Physics Letters, vol. 91, no. 6, Article ID 063124, 2007.

[56] C. Casiraghi, A. Hartschuh, E. Lidorikis et al., "Rayleigh imaging of graphene and graphene layers," Nano Letters, vol. 7, no. 9, pp. 2711-2717, 2007.

[57] Y. Li, S. Dai, J. John, and K. R. Carter, "Superhydrophobic surfaces from hierarchically structured wrinkled polymers," ACS Applied Materials \& Interfaces, vol. 5, no. 21, pp. 1106611073, 2013.

[58] U. Manna, M. C. D. Carter, and D. M. Lynn, “"shrink-to-fit” superhydrophobicity: Thermally-induced microscale wrinkling of thin hydrophobic multilayers fabricated on flexible shrinkwrap substrates," Advanced Materials, vol. 25, no. 22, pp. 30853089, 2013.

[59] H. Sojoudi, J. Baltazar, C. Henderson, and S. Graham, "Impact of post-growth thermal annealing and environmental exposure on the unintentional doping of CVD graphene films," Journal of Vacuum Science \& Technology B, Nanotechnology and Microelectronics: Materials, Processing, Measurement, and Phenomena, vol. 30, no. 4, Article ID 041213, 2012.

[60] J. Baltazar, H. Sojoudi, S. A. Paniagua et al., "Photochemical doping and tuning of the work function and dirac point in graphene using photoacid and photobase generators," Advanced Functional Materials, vol. 24, no. 32, pp. 5147-5156, 2014.

[61] D. A. C. Brownson, P. J. Kelly, and C. E. Banks, "In situ electrochemical characterisation of graphene and various carbonbased electrode materials: An internal standard approach," RSC Advances, vol. 5, no. 47, pp. 37281-37286, 2015.

[62] D. Cohen-Tanugi and J. C. Grossman, "Mechanical strength of nanoporous graphene as a desalination membrane," Nano Letters, vol. 14, no. 11, pp. 6171-6178, 2014.

[63] J. Groenewold, "Wrinkling of plates coupled with soft elastic media," Physica A: Statistical Mechanics and its Applications, vol. 298, no. 1-2, pp. 32-45, 2001.

[64] C. J. Shearer, A. D. Slattery, A. J. Stapleton, J. G. Shapter, and C. T. Gibson, "Accurate thickness measurement of graphene," Nanotechnology, vol. 27, no. 12, Article ID 125704, 2016. 


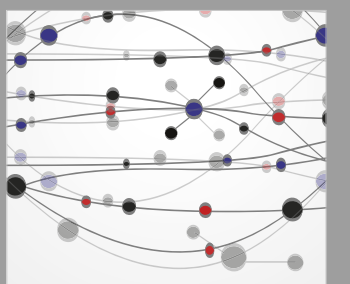

The Scientific World Journal
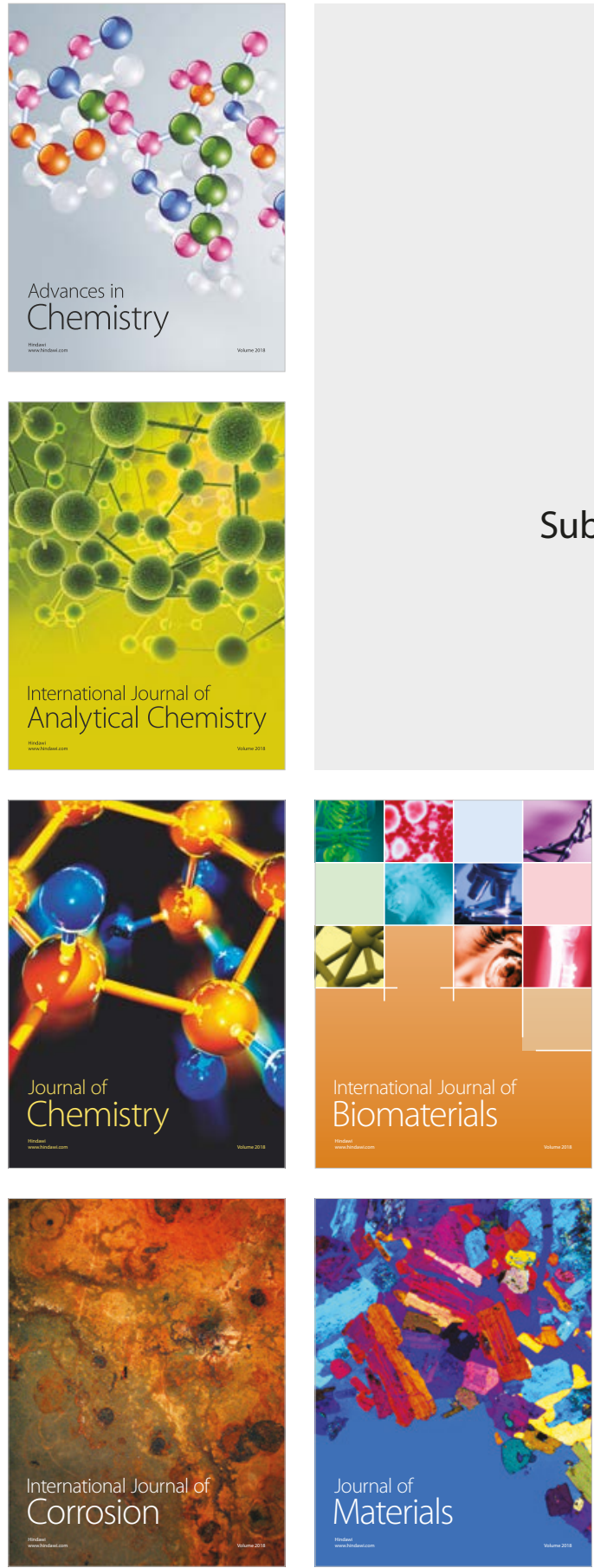

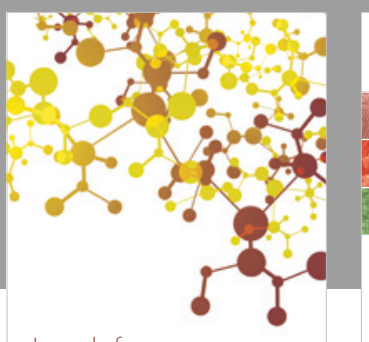

Journal of

Applied Chemistry
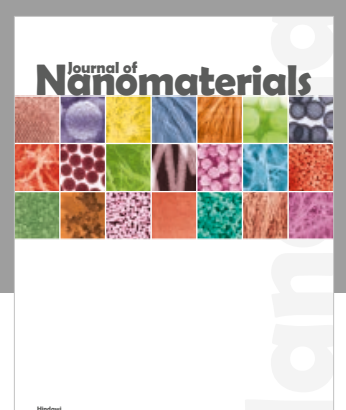

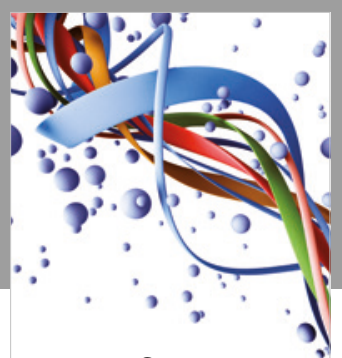

Scientifica

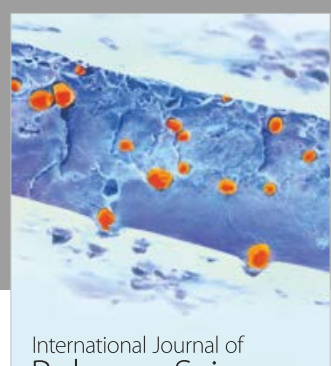

Polymer Science

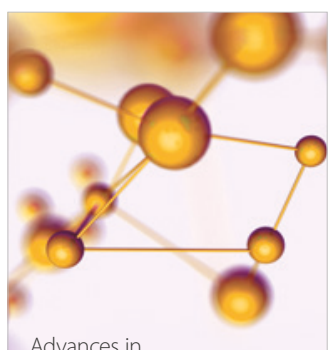

Physical Chemistry
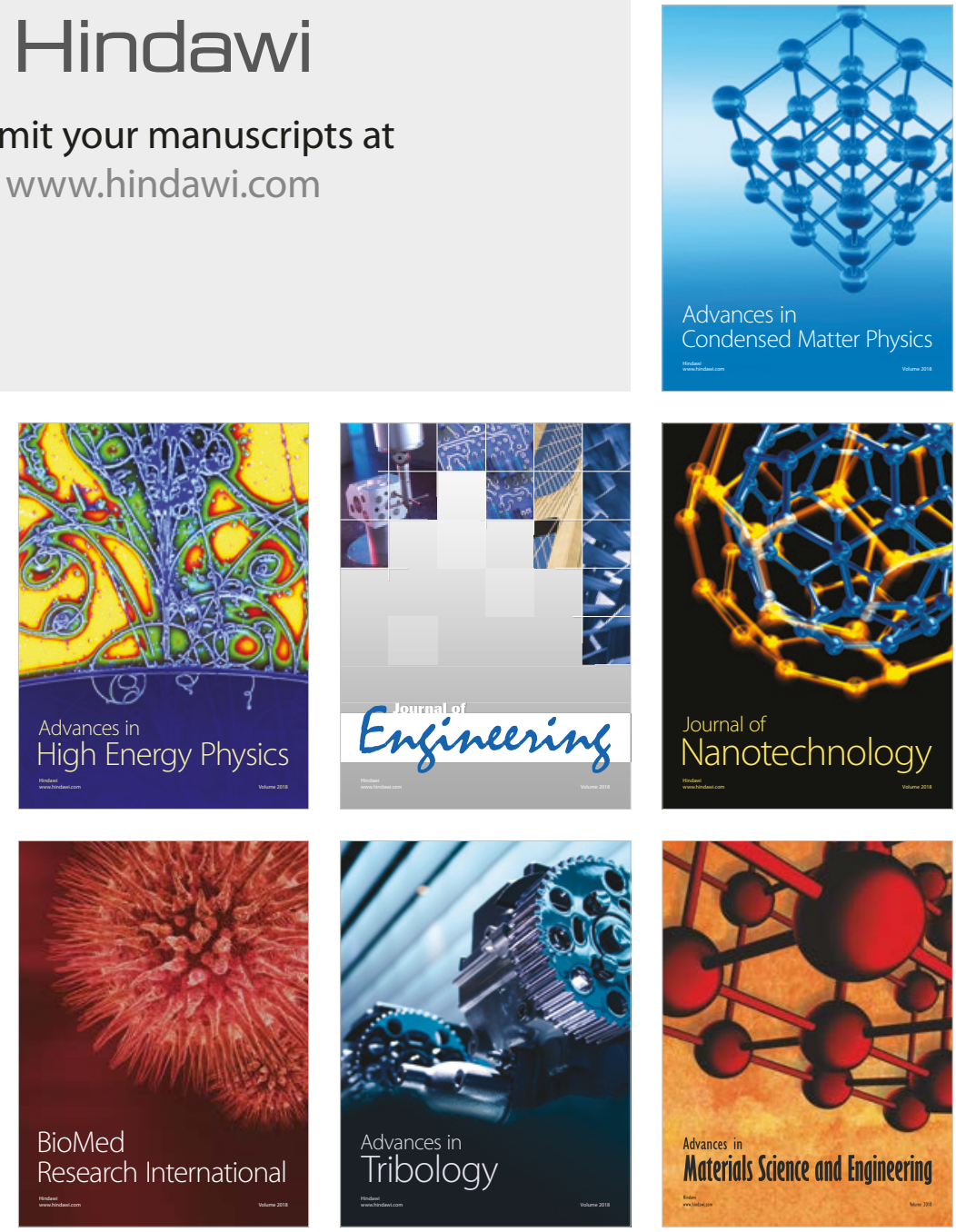